\title{
The science of negative index materials
}

\author{
Costas M Soukoulis ${ }^{1,2}$, Jiangfeng Zhou ${ }^{1}$, Thomas Koschny ${ }^{1}$, \\ Maria Kafesaki ${ }^{2}$ and Eleftherios $\mathbf{N}$ Economou $^{3}$ \\ ${ }^{1}$ Ames Laboratory and Department of Physics and Astronomy, Iowa State University, Ames, \\ IA 50011, USA \\ ${ }^{2}$ Institute of Electronic Structure and Lasers-FORTH, and Department of Materials Science \\ and Technology, University of Crete, Heraklion, Crete, Greece \\ ${ }^{3}$ Institute of Electronic Structure and Lasers-FORTH, and Department of Physics, \\ University of Crete, Heraklion, Crete, Greece
}

Received 29 November 2007, in final form 10 January 2008

Published 8 July 2008

Online at stacks.iop.org/JPhysCM/20/304217

\begin{abstract}
Metamaterials are designed to have structures that make available properties not found in Nature. Their unique properties (such as negative index of refraction, $n$ ) can be extended from $\mathrm{GHz}$ all the way to optical frequencies. We review the scaling properties of metamaterials that have been fabricated and give negative $n$ and negative permeability, $\mu$. It is found that most of the experimentally realized metamaterials have $\lambda / a$ between 2 ( $\mathrm{THz}$ and optical region) and 12 (GHz region), where $\lambda$ is the operation wavelength and $a$ is the size of the unit cell. The transmission losses for the experimental structures and the ratio $\lambda / a$ for the simulated structures are presented. Finally, a comparison of the different metamaterial designs (fishnet, cut and/or continuous wires, and split-ring resonators and wires) is given.
\end{abstract}

(Some figures in this article are in colour only in the electronic version)

\section{Introduction}

The concept of left-handed ( $\mathrm{LH}$ ) materials, where the electric field $(\mathbf{E})$, magnetic field $(\mathbf{B})$, and wavevector $(\mathbf{k})$ form a lefthanded coordinate system, was developed by Veselago [1] decades ago. These left-handed materials, which are also called negative index materials, have negative electrical permittivity $(\varepsilon)$, negative magnetic permeability $(\mu)$, and negative index of refraction $(n)$ at a common frequency band. However, it was only recently that such materials were investigated experimentally [2,3]. This was mainly thanks to the efforts of Professor Pendry, who was instrumental in suggesting artificial designs [4] that were able to give negative $\mu$. Although it has been well known how to obtain a $\varepsilon<0$ material easily (e.g. using wire arrays [5]), the realization of $\mu<0$ response (especially at high frequencies) has been a challenge, due to the absence of naturally occurring magnetic materials with negative $\mu$. The possibility of the realization of a $\mu<0$ material was predicted in 1999 by Pendry et al, who suggested a design made of concentric metallic rings with gaps, called split-ring resonator (SRR), which exhibits a $\mu<0$ regime in the vicinity of a magnetic resonance frequency [4]. The first experimental realization of LH-materials was achieved soon after [6], by separately constructing $\varepsilon<0$ and $\mu<0$ components, and then by combining them together.
In the past few years there has been ample proof for the existence of negative index materials (NIMs) in the $\mathrm{GHz}$ frequency range. A lot of groups were able to fabricate [6-30] NIMs with an index of refraction $n=-1$ with losses of less than $1 \mathrm{~dB} \mathrm{~cm}^{-1}[11,24]$. Recently different groups observed indirectly [12-15] negative $\mu$ at the $\mathrm{THz}$ region. In most of the $\mathrm{THz}$ experiments $[12,14,15]$ only one layer of SRRs was fabricated on a substrate and the transmission, $T$, was measured only for propagation perpendicular to the plane of the SRRs, exploiting the coupling of the electric field to the magnetic resonance of the SRR via asymmetry [31]. This way it is not possible to drive the magnetic permeability negative. Also, no negative $n$ with small imaginary part has been observed yet at the $\mathrm{THz}$ region $[20,21]$ in SRRs-based systems. One reason is that it is very difficult to measure with the existing topology of SRRs and continuous wires both the transmission, $T$, and reflection, $R$, along the direction parallel to the plane of the SRRs. Thus there is a need for alternative, improved and simplified designs that can be easily fabricated and experimentally characterized. This was recently achieved in the $\mathrm{GHz}$ region $[26,27]$, in the $\mathrm{THz}$ region $[20,21]$ and in the visible region $[22,23]$ by the use of pairs of finite length wires (cut wires) and the fishnet topology [16, 20, 21].

In this paper we will present results for the magnetic response of metamaterials for different frequency regions, as well as results on structures that were fabricated and show 
a negative index of refraction. Most of the left-handed structures at the $\mathrm{GHz}$ frequency region are based on the splitring resonators (SRRs) and continuous-wires design that was first proposed by Pendry et al [4], and fabricated by Smith et al [6]. To obtain negative $n$ at higher frequencies alternative designs were essential, such as cut and continuous wires, as well the fishnet design. The fishnet design has managed to push the frequency that gives negative index of refraction all the way to the visible spectrum. We have summarized all the experimental results that give negative $\mu$ and negative $n$ for all the frequency regions and we have plotted the operation (magnetic resonance) wavelength divided by the size of the unit cell $(\lambda / a)$ versus frequency. The ratio $\lambda / a$ in the experimental structures is higher at $\mathrm{GHz}$ frequencies (of the order of 10) than at $\mathrm{THz}$ frequencies (of the order of 2). This means that as one increases the frequency the metamaterials cannot be described well by an effective medium approach, because $\lambda / a$ is too small. We have also plotted the transmission losses, as $\mathrm{dB} / \lambda$, versus frequency for all the experimental structures that have shown negative $n$. The results show that losses are getting larger as the frequency increases. Finally, we have presented new numerical results for the magnetic resonance frequency for SRRs, cut wires, cut and continuous wires and fishnet design.

\section{Frequency dependence of the magnetic response of metamaterials}

Although the SRR is well suited as a local magnetic resonator in metamaterials, and can be scaled (although with limitations) up to near-optical frequencies [32], it has the disadvantage that by itself it does not provide a negative refractive index. To obtain a negative refractive index $n$ we need simultaneously negative values of the effective permeability $\mu(\omega)$ and permittivity $\varepsilon(\omega)$; hence a simultaneous negative magnetic and electric response is needed. In the microwave region a negative permittivity is usually achieved by combining the SRRs with an array of thin continuous wires, which provide a plasmonic $\varepsilon(\omega)$ with effective plasma frequencies in the microwave region [6-11, 36-39]. This approach is not well suited for $\mathrm{THz}$ frequencies because of dimensional problems and the absence of good conductors [32]. An alternative approach could be to exploit also the electric response of the SRR: the SRR itself has been shown to possess [33] an electric, Lorentz-type resonance (analog to that of a short piece of straight wire) that can provide a negative effective $\varepsilon(\omega)$ similarly to the resonant-negative $\mu(\omega)$ of the magnetic resonance. This electric resonant response is much stronger than the magnetic response; however, due to the principal geometry of the SRR it (almost) always occurs well above the magnetic resonance frequency, $\omega_{\mathrm{m}}$, such that there is virtually no chance to make the negative $\mu(\omega)$ and $\varepsilon(\omega)$ regions to overlap. (Because the $\varepsilon(\omega)$ resonance is much stronger and the $\varepsilon<0$ region therefore wider, we would have to move the $\mu(\omega)$ resonance into the negative permittivity region of the electric resonance.)

Alternatively to the SRR, a pair of parallel short wires (cut wires) can be used as a magnetic resonator [18, 19]. This geometry can be viewed as an extreme case of a two-gap
SRR ring [34] shrunk along the direction of the gaps. This limiting case of the two-gap SRR in principle can show both magnetic (resonant, circular, 'ring-like' current) and electric (resonant current, parallel in both wires) resonance; because of the greatly reduced loop inductance and the, own to the two gaps, larger capacitance, here $\omega_{\mathrm{m}}$ increases and can move very close to the electric resonance frequency, $\omega_{\mathrm{e}}$.

Like the SRR, the parallel short wires can be considered as an LC circuit; the inductive regime is the regime between the two wires (plus the mutual inductance and, at upper $\mathrm{THz}$ frequencies, the contribution from the electron mass), the capacitive regimes are the two inter-wire regimes close to the edges of the wires (along the $\mathbf{E}$ direction). If this basic resonator element is arranged in a periodic array to form a metamaterial, we have additional coupling between adjacent unit cells along the $\mathbf{E}$ direction, which becomes larger as pairs of adjacent unit cells become closer. Qualitatively, we can distinguish two limiting cases, which are shown in figure 1 (left) and figure 1 (right). Cut-wire-based designs have the advantage over SRR-based metamaterials that magnetic response and a negative refractive index can be achieved for normal incidence to the layered structure [34]. For SRRbased designs propagation in the SRR plane is required. This becomes a problem for high frequencies where layered structures are readily available to the experiment but true volume metamaterials are very complicated to achieve.

In the case of separate cut-wire pairs, as shown in figure 1 (left), the cut wires couple in the longitudinal (along E) direction capacitively. This capacitive coupling can be used to shift the electric resonance frequency relative to the magnetic resonance and make the negative electric and magnetic responses to overlap, so as to achieve a negative effective index. Metamaterials made of a single double-layer of cut-wire pairs have been fabricated and demonstrated to have resonant magnetic response in the upper $\mathrm{THz}$ region $[18,19]$.

Although the reduction to a very simple design providing both magnetic and electric resonant response simplifies fabrication, these structures have the tendency to have huge losses, attributed in part to employing two resonances with the associated resonant losses. An alternative coupling of the cut-wire pairs in a periodic metamaterial is to introduce 'thin' interconnects between the cut wires of adjacent unit cells in the longitudinal direction, hence facilitating an inductive instead of a capacitive coupling. Conveniently, the width of the cut wires in the $\mathbf{H}$ direction can be extended across the unit cell to enhance the magnetic response (volume filling factor). The result of this operation is the fishnet structure shown in figure 1 (right). A detailed study [35] has been performed for different versions of the fishnet design. Here, the wide block $w_{x} \times a_{y}$ forms a short-wire pair, the thin bridges in the E-direction are the inductive interconnects. This structure exposes the magnetic resonant response of the cut-wire pairs (here as anti-parallel currents in the E-direction in the wide blocks) but the (lowest) electric resonance is replaced by an electric plasmonic response due to the replacement of the longitudinal coupling capacitance by a coupling inductance. As a simple image, one may imagine this as a combination of cut-wire pairs (magnetic resonance) with continuous wires (providing the 

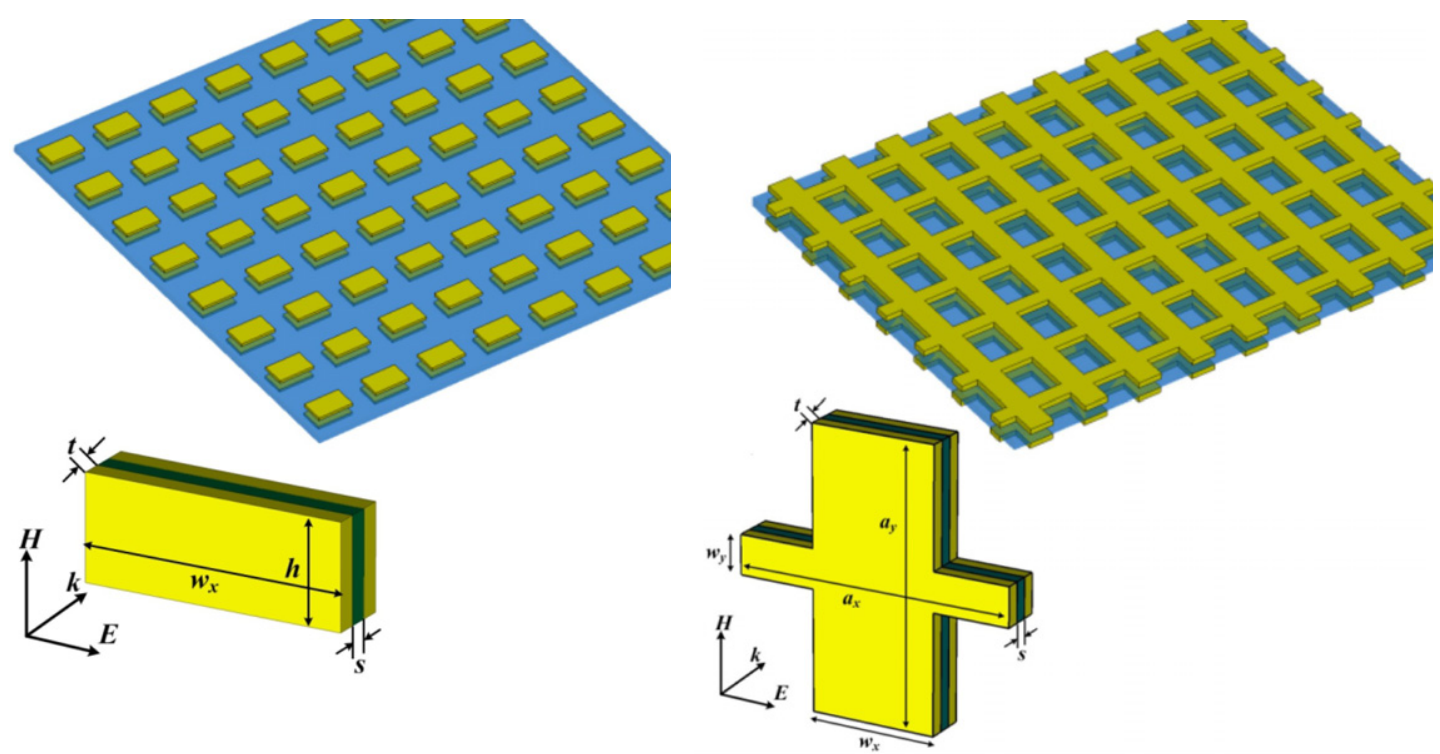

Figure 1. Geometries for cut-wire pair arrays (left panel) and the fishnet structure (right panel). Both consist of a patterned metallic double-layer (yellow outer layer, usually $\mathrm{Au}$ ) separated by a thin dielectric (blue inner layer).

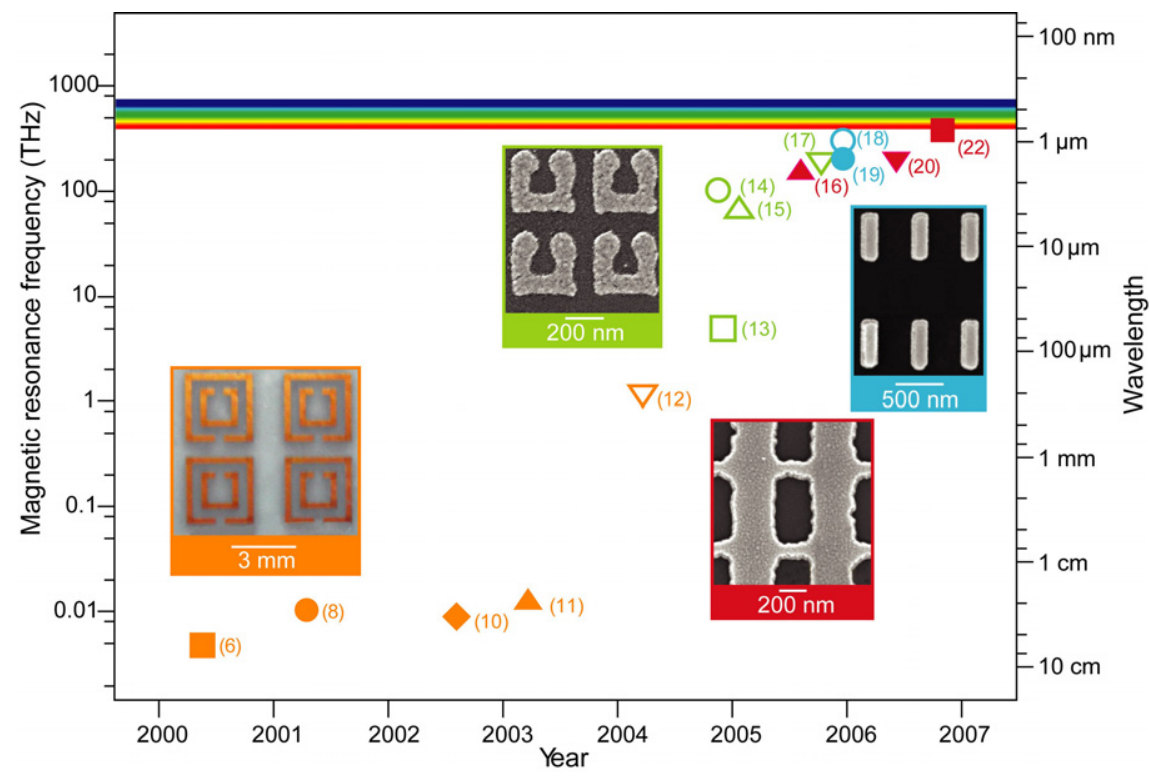

Figure 2. The progress in the negative permeability, $\mu$, and negative index, $n$, materials development in the last seven years. The solid symbols denote $n<0$; the open symbols denote $\mu<0$. Orange: data from structures based on the double split-ring resonator (SRR); green: data from U-shaped SRR; blue: data from pairs of metallic cut-wire pairs; red: data from the 'fishnet' structure. The four insets give pictures of fabricated structures in different frequency regions. Reprinted with permission from [2]. Copyright (2007) AAAS.

electric plasmonic response) [26, 27]. The 'missing' electric resonance tends to reduce the losses in this case.

In figure 2 we present the progress in the development of negative magnetic permeability and negative refractive index materials over the last seven years. In the early years (20002003) the design of choice for the achievement of a negative $\mu$ was the SRR, introduced by Pendry. To obtain a negative $n$, continuous wires were arranged next to the SRRs. As one can see from figure 2, double ring SRR was used in the original experiments, but as the frequency was getting larger the one ring SRR was used to obtain negative $\mu$, because of ease of fabrication. In 2004 a negative $\mu$ at 1, 6 and $100 \mathrm{THz}$ was obtained by scaling down the SRR structures [12-14]. The scaling down of the SRRs has given negative $\mu$ up to $200 \mathrm{THz}$ [17].

Although the SRRs and wires design is very useful at $\mathrm{GHz}$ frequencies where the sizes of the SRRs are of the order of $1 \mathrm{~cm}$, and it is relatively easy to form multi-stacks and to propagate along the plane of the SRRs (so as to have the magnetic field perpendicular to the plane of the SRRs and the electric field parallel to the wires, and thus to be able to obtain negative $n$ response), it is not convenient at optical frequencies. 


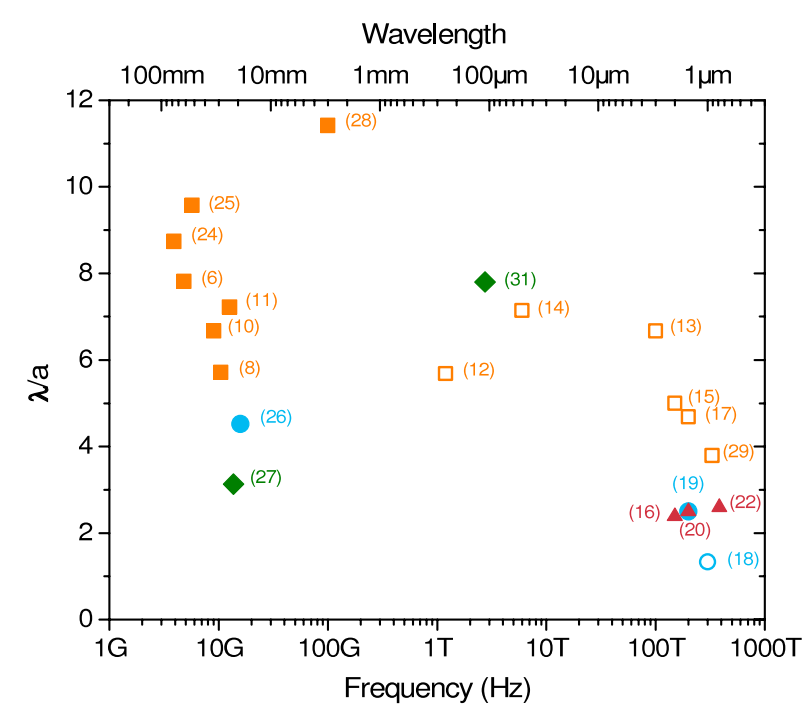

Figure 3. The ratio $\lambda / a$ versus frequency for all the existing experimental results that give negative $n$ and/or negative $\mu$. The solid symbols denote $n<0$; the open symbols denote $\mu<0$. The number next to the symbols gives the reference of the experimental paper. Orange (solid and hollow squares): data from structures based on the double split-ring resonator (SRR); green (solid diamonds): data from U-shaped SRR; blue (solid and hollow circles): data from pairs of metallic cut wires; red (solid triangles): data from the 'fishnet' structure.

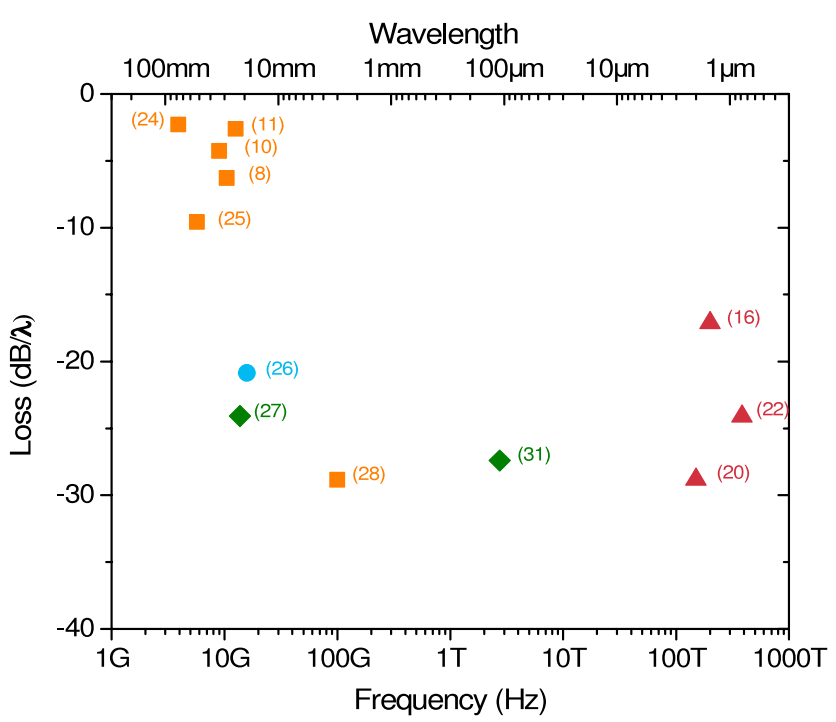

Figure 4. Transmission loss, in units of $\mathrm{dB} / \lambda$ versus frequency for all the existing experimental structures that show a negative $n$. The number next to the symbols gives the reference of the experimental paper. Orange (solid squares): data from structures based on the double split-ring resonator (SRR); green (solid diamonds): data from U-shaped SRR; blue (solid circles): data from pairs of metallic cut wires; red (solid triangles): data from the 'fishnet' structure.

So there was a need to find alternative designs that will be used to give negative $\mu$ and negative $n$ at micron and visible frequencies. As one can see from figure 2, the new designs (cut wires and fishnet) that were independently realized $[16,18,19]$ and published in 2005 manage to give negative $n$ all the way to the visible frequencies $[22,23]$. Only 7 years after their first demonstration, negative index materials have been brought from microwave frequencies toward the visible regime. The ideas for potential applications, such as superlensing [40, 41] and cloaking $[42,43]$, that Professor Pendry has put forward, can become a reality with the fast development of NIMs. Moreover, Professor Pendry has introduced a new route to obtain [44] a negative $n$, that of chirality. In order to meet though the proposed applications one needs to reduce the losses of NIMs, as will be discussed below.

One important difference between negative index materials and photonic crystals is that the ratio $\lambda / a$ is much larger in NIMs than in photonic crystals. In metamaterials or in NIMs the wavelength of operation is due to the resonance of the localized 'magnetic' structures (SRRs or cut wires), and can be tuned to be much higher than the size of the unit cell, while at photonic crystals the operation frequency that gives a photonic gap is due to Bragg scattering, giving $\lambda / a$ of the order of one. So the metamaterials or NIMs can really be described by an effective medium theory. In figure 3 we examine that, presenting experimental results for $\lambda / a$ versus frequency for all the fabricated metamaterials structures, ranging from $\mathrm{GHz}$ all the way to visible frequencies. It is very interesting to note that $\lambda / a$ for $\mathrm{GHz}$ frequencies has a range of 6-12, with the majority of the data to be around 8 . Thus, the homogeneous effective medium theory can be applied for the $\mathrm{GHz}$ structures and the retrieval procedure $[45,46]$ that gives the effective $\varepsilon, \mu$ and $n$ of those structures is correct. However, as the frequency increases and approaches the $\mathrm{THz}$ region, the ratio $\lambda / a$ decreases and reaches a value of 2 . So it is very difficult to argue that the metamaterials at $\mathrm{THz}$ and visible frequencies can be described by an effective medium theory; there is a need to find new designs or new ways to improve the ratio of $\lambda / a$ at $\mathrm{THz}$ and visible frequencies.

Another crucial parameter for the operation of a metamaterial is the losses. We have made an effort to quantify the transmission loss of the existing metamaterials that possess negative index of refraction. In figure 4 , we present the transmission loss in units of $\mathrm{dB} / \lambda$ versus frequency. One can see clearly that the loss at low frequencies is small (of the order of $1-5 \mathrm{~dB} / \lambda$ ), while as the frequency increases the loss is getting bigger, approaching values of almost $30 \mathrm{~dB} / \lambda$. The loss here has been calculated from the experimental transmission values, which have been measured for a given structure thickness, and by assuming an exponential length dependence of the transmission. Of course this implies the assumption that the transmission drop is due to the loss and not to the reflection. So the numbers of figure 4 are upper limit of the losses. If the reflection, $R$, was also measured, we could have calculated the effective transmission, which is defined as $T /(1-R)$ and the losses given in figure 4 could be reduced. Even with this additional reduction the losses are really high, especially at $\mathrm{THz}$ and visible frequencies. So one needs to find ways to reduce them at high frequencies, or to find applications that are not affected by the large losses, such as applications employing thin metamaterial slabs.

As was discussed above, in connection with figure 3, the experimentally measured ratio of $\lambda / a$ of metamaterials decreases as the magnetic resonance frequency of the 


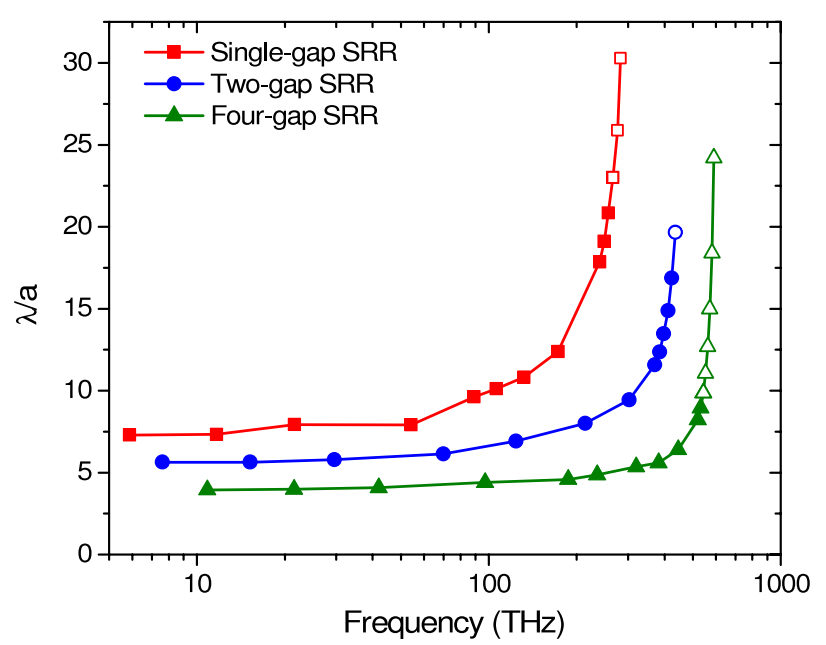

Figure 5. $\lambda / a$ ratio of single gap, two gap and four gap SRRs using simulated data shown in [32]. The solid symbols show magnetic resonances with negative $\mu$. As the resonance frequencies increase, the resonances are getting weaker, and $\mu$ cannot reach negative values anymore, which are shown as hollow symbols.

'magnetic' structures increases. This behavior is completely opposite to the one predicted theoretically for SRRs, and presented in figure 5 . In figure 5 we have plotted the calculated ratio $\lambda / a$ versus the magnetic resonance frequency for SRRs of one, two and four gaps. It can be seen that as the resonance frequency of the SRRs increases the ratio of $\lambda / a$ remains constant, all the way to $100 \mathrm{THz}$ for the single gap SRRs. This means that as the size of the unit cell of the SRR decreases the wavelength decreases proportionally, and we therefore have an almost perfect scaling. However, for higher frequencies (higher than $100 \mathrm{THz}$ for the single gap SRR), as the resonance frequency increases, which means that the size of the unit cell decreases, the ratio of $\lambda / a$ increases dramatically. This behavior is due to the fact that the scaling of the resonance frequency breaks down in small length scales, where as the size of the unit cell decreases the resonance wavelength saturates to a constant value. This saturation was explained very well in [32] and it originates from the fact that at small SRR unit cell sizes the kinetic energy of the moving electrons becomes comparable to the energy stored in the magnetic field. So far, all the experimental results though were not able to reproduce the numerical results shown in figure 5. This might be due to the fact that in smaller length scales the experimental limitations (in terms of fabrication) have led to designs of higher and higher capacitance, which push the magnetic resonance wavelength to higher values and do not allow to figure out and examine the scaling properties of a single design.

In figure 6 we present numerical results of the ratio $\lambda / a$ versus the magnetic resonance frequency for the fishnet design, the cut wires and the cut and continuous wires design. The behavior shown in figure 6 is in agreement with the results presented in figure 5 for the SRRs. At low frequencies we have scaling, where $\lambda / a$ is constant, while at high frequencies $\lambda / a$ increases dramatically. In figure 6 we also plot $\lambda / a$ for only cut wires. As was discussed extensively $[18,27,34]$ in the literature, it is very difficult to obtain both $\varepsilon$ and $\mu$ to be negative and therefore negative $n$ at the same frequency region with only cut wires. If we add the continuous wires next to the cut wires, one can easily obtain negative $n$. Another very important observation is that for the same frequency the ratio $\lambda / a$ for the fishnet design is almost a factor of 2 lower than that of the cut and continuous wires design. The higher $\lambda / a$ for the fishnet is due to the strong inductive coupling between the adjacent unit cells along $\mathbf{E}$ direction, which significantly reduces the total inductance of the design (compared to the cut-wires inductance), leading to a larger magnetic resonance frequency (smaller magnetic resonance wavelength) for a given unit cell size. Both the fishnet [16, 20-22] and cut and continuous wires design [27] give negative $n$. It is therefore very important to use the cut and continuous wire design at high frequencies if one needs to describe the NIMs as an

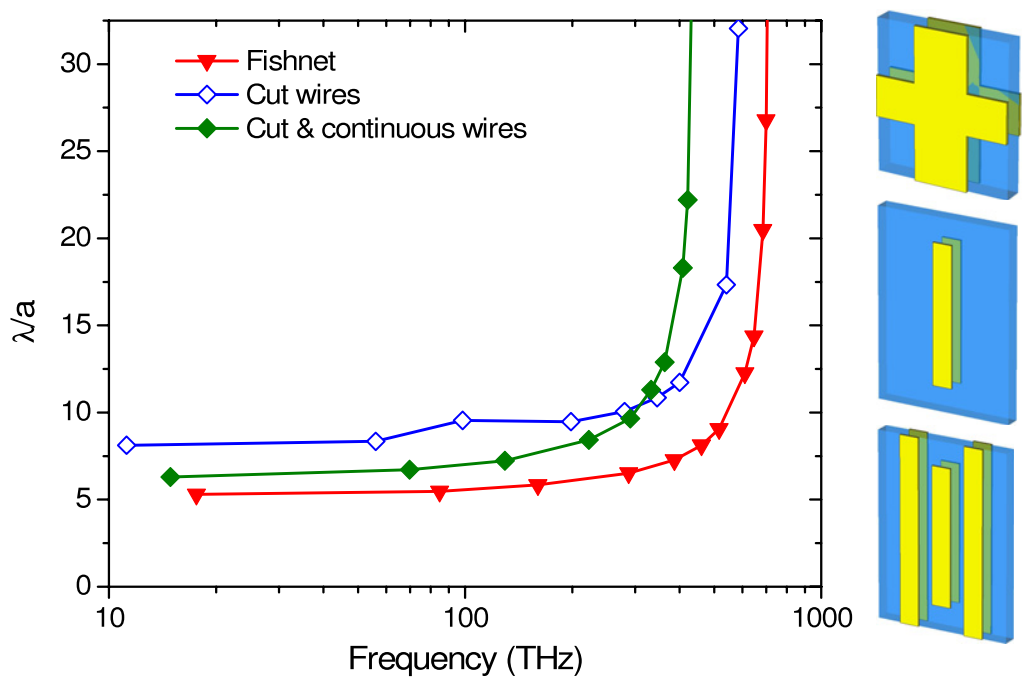

Figure 6. Left panel: simulated $\lambda / a$ ratio versus magnetic resonance frequency for the structures shown in the right panel, i.e. fishnet (top-right), pairs of cut wires (middle-right) and cut and continuous wires (bottom-right). Right panels show one unit cell of the fishnet, cut wires and cut and continuous designs respectively. 
effective medium. This might be useful if one wants to use the metamaterials for superlenses applications at $\mathrm{THz}$ frequencies. The reason is that $\lambda / a$ for cut and continuous wires is much larger than that of fishnet.

\section{Conclusions}

In conclusion, we have reviewed the status of the design, fabrication and characterization of metamaterials that give a negative index of refraction. In addition we have presented results for the ratio of the wavelength over the size of the unit cell size versus frequency for different designs (SRRS, cut wires, cut wires and continuous wires and fishnet) that give a negative $\mu$. Only 7 years after the first demonstration, negative index metamaterials have been brought from microwaves to the visible regime. Our detailed studies have shown that one of the main difficulties in the further development of metamaterials is the existence of strong losses, especially in the infrared and visible regime. So there is a need for finding new designs or ways to reduce the losses. We are now in position to move forward and further develop the materials and methods that will make these novel materials useful for applications as were envisioned by Professor Sir John Pendry, the founder of the metamaterials field.

\section{Acknowledgments}

Work at the Ames Laboratory was supported by the Department of Energy (Basic Energy Sciences) under Contract No. DE-AC02-07CH11358. This work was partially supported by the AFOSR under MURI grant (FA9550-06-1-0337), by DARPA (Contract No. MDA-972-01-2-0016), Office of Naval Research (Award No. N00014-07-1-0359) and EU projects: Metamorphose and PHOREMOST, and Molecular Imaging (LSHG-CT-2003-503259).

\section{References}

[1] Veselago V G 1968 Sov. Phys.-Usp. 10509

[2] For recent reviews see Soukoulis C M, Kafesaki M and Economou E N 2006 Adv. Mater. 181941

Soukoulis C M, Linden S and Wegener M 2007 Science 31547

[3] Shalaev V M 2007 Nat. Photon. 141

[4] Pendry J, Holden A, Robbins D and Stewart W 1999 IEEE Trans. Microw. Theory Tech. 472075

[5] Pendry J B, Holden A, Stewart W and Youngs I 1996 Phys. Rev. Lett. 764773

Smith D R, Schultz S, Kroll N, Sigalas M, Ho K M and Soukoulis C M 1994 Appl. Phys. Lett. 65645

[6] Smith D R, Padilla W, Vier D, Nemat-Nasser S and Schultz S 2000 Phys. Rev. Lett. 844184

[7] Shelby R A, Smith D R, Nemat-Nasser S C and Schultz S 2001 Appl. Phys. Lett. 78489

[8] Shelby R A, Smith D R and Schultz S 2001 Science 29277

[9] Parazzoli C G, Greegor R B, Li K, Koltenbah B E C and Tanielian M 2003 Phys. Rev. Lett. 90107401

[10] Bayindir M, Aydin K, Ozbay E, Markos P and Soukoulis C M 2002 Transmission properties of composite metamaterials in free space Appl. Phys. Lett. 81120

[11] Greegor R B et al 2003 Appl. Phys. Lett. 822356

[12] Yen T J, Padilla W J, Fang N, Vier D C, Smith D R, Pendry J B, Basov D N and Zhang X 2004 Science 3031494
[13] Katsarakis N, Konstantinidis G, Kostopoulos A, Penciu R, Gundogdu T, Kafesaki M, Economou E, Koschny T and Soukoulis C M 2005 Opt. Lett. 301348

[14] Linden S, Enkrich C, Wegener M, Zhou J F, Koschny T and Soukoulis C M 2004 Science 3061351

[15] Zhang S et al 2005 Phys. Rev. Lett. 94037402

[16] Zhang S et al 2005 Phys. Rev. Lett. 95203901

[17] Enkrich C, Wegener M, Linden S, Burger S, Zschiedrich L, Schmidt F, Zhou J F, Koschny T and Soukoulis C M 2005 Phys. Rev. Lett. 95203901

[18] Dolling G, Enkrich C, Wegener M, Linden S, Zhou J and Soukoulis C M 2005 Opt. Lett. 303198

[19] Shalaev V M et al 2005 Opt. Lett. 303356

[20] Dolling G, Enkrich C, Wegener M, Soukoulis C M and Linden S 2006 Opt. Lett. 311800

[21] Dolling G, Enkrich C, Wegener M, Soukoulis C M and Linden S 2006 Science 312892

[22] Dolling G, Wegener M, Soukoulis C M and Linden S 2007 Opt. Lett. 3253

[23] Chettiar U K, Kildishev A V, Yuan H-K, Cai W, Xiao S, Drachev V P and Shalaev V M 2007 Opt. Lett. 321671

[24] Aydin K, Guven K, Kafesaki M, Zhang L, Soukoulis C M and Ozbay E 2004 Opt. Lett. 292623

[25] Katsarakis N, Kafesaki M, Tsiapa I, Economou E N and Soukoulis C M 2007 Photon. Nanostruct.-Fundam. Appl. 5149

[26] Zhou J F, Koschny T, Zhang L, Tuttle G and Soukoulis C M 2006 Appl. Phys. Lett. 88221103

[27] Zhou J F, Zhang L, Tuttle G, Koschny T and Soukoulis C M 2006 Phys. Rev. B 73041101

[28] Gokkavas M, Guven K, Bulu I, Aydin K, Kafesaki M, Penciu R, Soukoulis C M and Ozbay E 2006 Phys. Rev. B 73193103

[29] Klein M W, Enkrich C, Wegener M, Soukoulis C M and Linden S 2006 Opt. Lett. 311259

[30] Gundogdu T F, Katsarakis N, Kafesaki M, Penciu R S, Konstantinidis G, Kostopoulos A, Economou E N and Soukoulis C M 2008 Opt. Express submitted

[31] Katsarakis N, Koschny T, Kafesaki M, Economou E N and Soukoulis C M 2004 Appl. Phys. Lett. 842943

[32] Zhou J F, Koschny T, Kafesaki M, Economou E N, Pendry J B and Soukoulis C M 2005 Phys. Rev. Lett. 95223902

[33] Koschny T, Kafesaki M, Economou E N and Soukoulis C M 2004 Phys. Rev. Lett. 93107402

[34] Zhou J, Economou E N, Koschny Th and Soukoulis C M 2006 Opt. Lett. 313620

[35] Kafesaki M, Tsiapa I, Katsarakis N, Koschny Th, Soukoulis C M and Economou E N 2007 Phys. Rev. B 75235114

[36] Soukoulis C M, Koschny Th, Zhou J, Kafesaki M and Economou E N 2007 Phys. Status Solidi b 2441181

[37] Penciu R, Kafesaki M, Gundogdu T F, Economou E N and Soukoulis C M 2006 Photon. Nanostruct.-Fundam. Appl. 412

[38] Gundogdu T F, Gokkavas M, Guven K, Kafesaki M, Soukoulis C M and Ozbay E 2007 Photon. Nanostruct.-Fundam. Appl. 5106

[39] Danithe H, Foteinopoulou S and Soukoulis C M 2006 Photon. Nanostruct.-Fundam. Appl. 4123

[40] Pendry J B 2000 Phys. Rev. Lett. 853966 Pendry J B and Smith D R 2006 Sci. Am. 29560

[41] Smith D R, Pendry J B and Wiltshire M C K 2004 Science 305788

[42] Pendry J B, Schurig D and Smith D R 2006 Science 3121780

Schurig D, Mock J J, Justice B J, Cummer S A, Pendry J B, Starr A F and Smith D R 2006 Science 314977 
[43] Guenneau S, Anantha Ramakrishna S, Enoch S, Chakrabarti S, Tayeb G and Gralak B 2007 Photon. Nanostruct.-Fundam. Appl. 563

[44] Pendry J B 2004 Science 3061353
[45] Smith D R, Schultz S, Markos P and Soukoulis C M 2002 Phys. Rev. B 65195104

[46] Koschny T, Markos P, Economou E N, Smith D R, Vier D C and Soukoulis C M 2005 Phys. Rev. B 71245105 